\title{
Fast Switching and Gain Control of Optical Repeater Units for Burst Mode Upstream Transmission Over Super-PONs
}

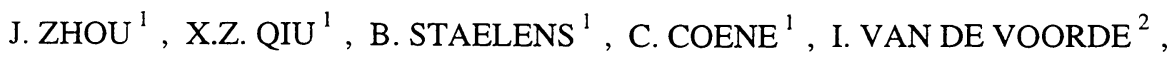 \\ J. VANDEWEGE ${ }^{1}$ \\ ${ }^{1}$ University of Gent, INTEC, Sint-Pietersnieuwstraat 41, B-9000 Gent, Belgium \\ ${ }^{2}$ Alcatel Telecom, F. Wellesplein 1, B-2018 Antwerpen, Belgium
}

\begin{abstract}
This paper presents a fast switching and gain control technique of optical repeater units (ORUs) for burst mode upstream transmission over a Super-PON system by using semiconductor optical amplifiers (SOAs). Measurement results confirm that switching of the SOA can be accomplished within 3 bits at $155.52 \mathrm{Mbps}$ and that the gain control range is more than $10 \mathrm{~dB}$.
\end{abstract}

Keywords: B-ISDN, SuperPON, TDM, TDMA, SOA, ASE, SNR, ORU, OLT.

\section{INTRODUCTION}

High capacity optical transmission systems are required to support broad-band Integrated Service Digital Networks (B-ISDNs) in the 21st century, which will provide universal communication channels for multimedia services including voice, video and data [1]. A lot of efforts have been made on Asynchronous Transfer Mode (ATM) transmission where data is grouped into cells. Recently a conventional Passive Optical Network (PON) is recognised as an efficient and economical solution for transparent ATM access networks. By introduction of passive optical splitters, a number of residential customers are able to share the same transmission network and line termination facilities. However, the commercial PON system capacity is currently limited from $50 \mathrm{Mbps}$ to $622 \mathrm{Mbps}$. A splitting factor of 16 or 32 over an optical fiber length of maximum $20 \mathrm{~km}$ is state of the art [2].

Thanks to the latest developments on Optical Amplifiers (OAs), a higher split ratio and a longer feeder length can be achieved to realise advanced, cost effective networks. This introduces a so-called Super-PON system which is able to support up to 2048 subscribers and a range of $100 \mathrm{~km}$ [2]. In this proposed SuperPON system, the system capacity is increased to $2.5 \mathrm{Gbps}$ TDM downstream and $311 \mathrm{Mbps}$ ATM-based TDMA upstream. This challenging task can be fulfilled by introducing optical amplifiers in specific fiber paths to compensate network losses. The crucial technical issues are being investigated in ACTS PLANET (Photonic Local Access NETwork) project, supported by European Commission. The objective of the PLANET project is to evaluate a high split ratio, wide range and cost-effective optical access network as shown in Fig. 1 [3].

Downstream communication is in single talker and continuous mode, therefore no fast gain setting is needed. This allows us to use low noise figure (NF) Erbium Doped Fiber Amplifiers (EDFAs) with high 
gain and high saturation power as optical amplifiers. For upstream transmission, 16 parallel optical amplifiers at the amplified splitter section must be switched on/off in nanoseconds in order to solve the noise funnelling problem due to Amplified Spontaneous Emission (ASE) noise, minimise the guard time and the overhead of the ATM cell. Short carrier lifetime SOAs are used with the penalty of a relatively high noise figure.

In the first lab-demonstrator, $1310 \mathrm{~nm}$ bi-directional Multiple Quantum Well (MQW) laser amplifiers CQF882/E from Philips are employed at the upstream Optical Repeater Units (ORUs). Using $1310 \mathrm{~nm}$ SOAs as optical amplifiers is a good choice for meeting the power budget requirement of the system, also for the switching capability and gain compression functions of the ORUs. It is planned to use an existing ATM-based FITL APON system, in which the upstream transmission is at $155.52 \mathrm{Mbps}$, to evaluate and optimise a reference architecture for a field trial of the Super-PON. The bit-rate will be upgraded to 311 Mbps in the field trial.

In this paper, we start with an analysis of characteristics of SOAs and introduce the necessity of fast switching and gain control on upstream transmission in section 2 . Then we present the implementation of SOA driver circuitry and a control mechanism for the ORUs in section 3 . The measurement results of the SOA module confirm that switching of the SOAs can be accomplished within 3 bits (19.2 ns) at 155.52 Mbps, and show a gain control range of more than $10 \mathrm{~dB}$.

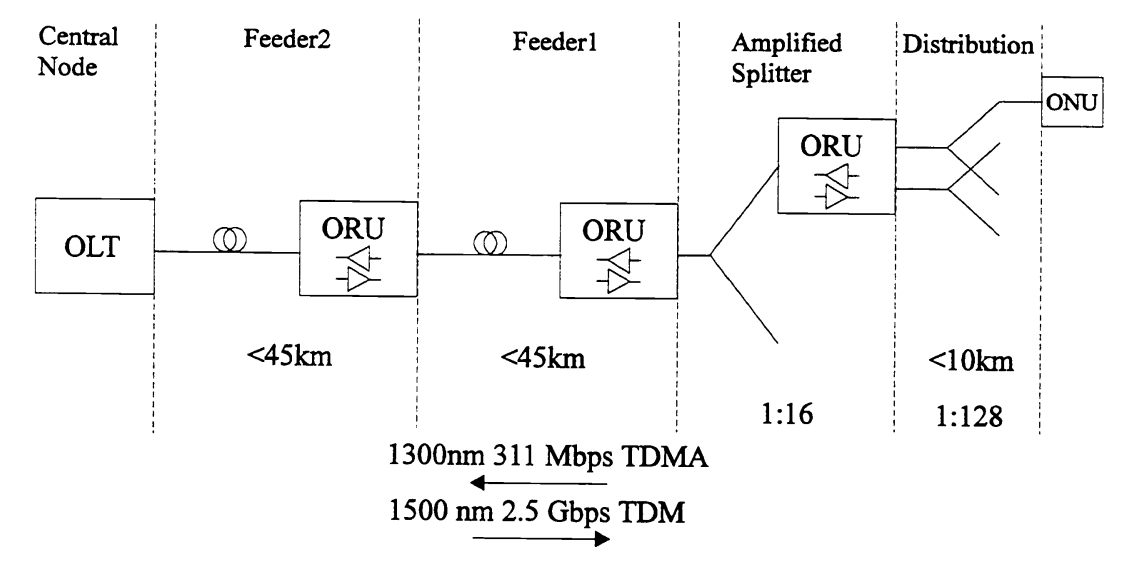

Fig. 1 Functional diagram of a Super-PON for bi-directional transmission

\section{SWITCHING AND GAIN CONTROL OF THE SOA}

Recent advancements in low noise, high gain and polarisation insensitivity of $1300 \mathrm{~nm}$ SOAs have brought them to high bit-rate lightwave systems with an advantage of minimum dispersion at $1300 \mathrm{~nm}$ [4]. By employing MQW active layers, the SOAs are significantly improved in high saturation output power and low noise figure, and can have a very short carrier recovery time of less than $1 \mathrm{~ns}$.

According to the PLANET architecture shown in Fig.1, at least 16 SOAs are required to operate in parallel at the amplified splitter section to obtain the high splitting factor of 2048. Consequently Signal-to- 
Noise Ratio (SNR) of the system is seriously degraded due to the accumulation of ASE noise of these 16 SOAs. The total ASE power at the output of an optical amplifier is given by [5]:

$$
P_{S P}=\int N_{S P}(G(v)-1) h v d v
$$

where, $G(v)$ is a gain profile of the SOA, $N_{S P}$ the spontaneous emission parameter, $h$ Planck's constant, and $v$ optical carrier frequency. The gain profile $G(v)$ can be assumed as a Gaussian-like function of the optical wavelength $\lambda$ with a typical $3 \mathrm{~dB}$ bandwidth of $50 \mathrm{~nm}$.

Fig. 2 shows the spectrum of ASE power of the SOA, which confirms the assumption of the gain profile. The measured total ASE power after the 16:1 combiner is depicted in Fig.3, where $\mathrm{N}$ is the number of SOAs in parallel. This high optical power will cause a severe saturation of the SOAs in the next sections, which compresses zero and one level of the data, and deteriorates SNR [6]. It is clear that SOAs should be switched off when they don't carry burst mode ATM cells. Fortunately, the feature of very short carrier lifetime of SOAs makes it possible to achieve fast switching at very precise moments.

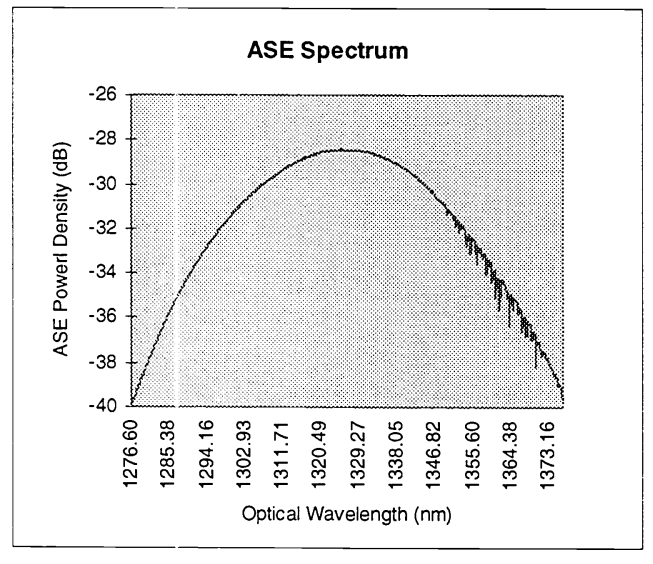

Fig.2 Measured ASE spectrum at center wavelength $1326.6 \mathrm{~nm}$ with $3 \mathrm{~dB} \mathrm{BW}$ of $51.2 \mathrm{~nm}$

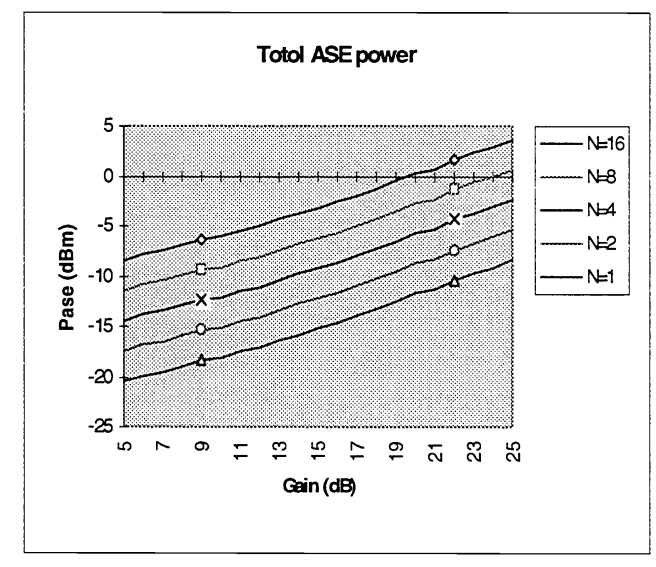

Fig.3 Total ASE power with N SOAs operating in parallel

On the other hand, dynamic power compression at the ORUs is needed to drastically reduce the dynamic range requirement of the burst-mode RX at the Optical Line Termination (OLT). The gain of SOAs must be controlled in order to compensate the differential loss at different sections of the upstream path in the extreme conditions of the best case and worst case. The differential optical power budget includes : optical power variations of transmitters at the ONUs, the differential loss of the optical networks, and the gain uncertainty of each SOA due to polarisation, wavelength and ageing. With a given SOA, variable optical gain can be achieved by setting different values of injection current. Fig.4 illustrates the gain curve of the SOA module versus injection current with two different input power levels as parameter, which indicates that more than $10 \mathrm{~dB}$ gain control range can be obtained if the SOA injection current varies 
from $50 \mathrm{~mA}$ to $150 \mathrm{~mA}$. Below $50 \mathrm{~mA}$, the slope of the gain curve is a little too steep for practical use, which will result in inaccurate control of the SOA gain.

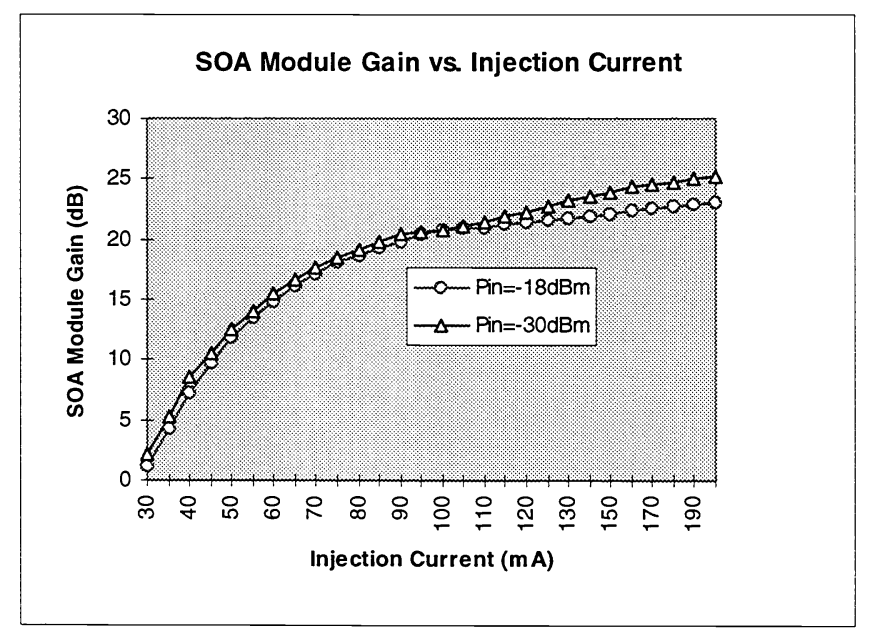

Fig. 4 SOA module gain vs. injection current

\section{IMPLEMENTATION AND MEASUREMENT RESULTS}

Fast switching and gain control of SOAs is accomplished by a feedforward and feedback control loop, which is functionally described in Fig.5. The feedforward control loop is baced on activity detection and power level measurement. The incoming cell is detected and the power level is measured by a Fortress controller unit (a FORTH-based real time control system). Using this measured input optical power as an entry, an optimum SOA injection current is selected from a RAM baced look-up table on a cell by cell basis. Once the output of DAC is stable, the SOA can be switched on by an on/off control signal. The feedback control loop monitors the output power of the SOA for compensating slow changing of the SOA characteristics due to ageing and temperature changing. If required the look-up table in the control systems will be updated.

A block diagram of the SOA driver circuit is depicted in Fig. 6. The electrical on/off control signal based on ATM cell is applied to a PECL line driver to switch a current source to either the SOA or a resistive load by using fast compound pairs of PNP transistors. The reason to employ PNP transistors is that the cathode of the SOA is connected to the case of the butterfly-type package. Although the switching speed is limited by the PNP transistors, an alternative solution would require an electrically floating package. This would deteriorate the switching speed and waveform due to additional parasitic capacitance between the package and the heatsink. An overshoot compensation circuit protects the SOA, and realises a smooth but fast turn on/off transition. A D/A convertor level setting circuit is used to set the different values of the current source. The temperature control circuit works as an "electrical bridge" to ensure the SOA chip working at a fixed temperature $\left(20^{\circ} \mathrm{C}\right)$ by supplying amount of current to a Peltier cooler inside the SOA package. 


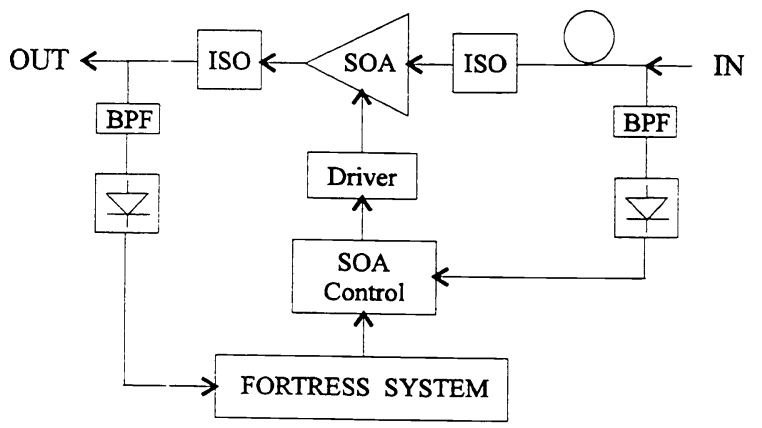

Fig.5 Functional diagram of SOA control

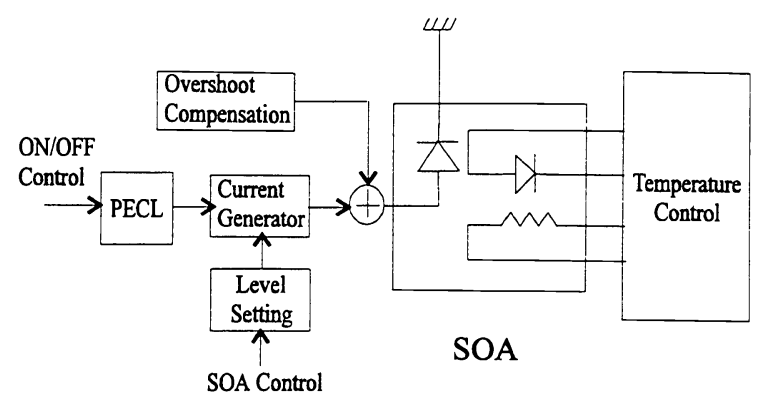

Fig.6 SOA driver with fast switching capability

Some measurement results are illustrated in the following figures. An example of the gain control is given in Fig.7. For a reason of good visibility, the injection current of the SOA is shifted between $150 \mathrm{~mA}$ and $100 \mathrm{~mA}$ at the rate of the ATM cells with fixed input power of $-30 \mathrm{dBm}$. It can be observed that the gain control range $\Delta G$ is $4.0 \mathrm{~dB}$, and that the DC offsets caused by ASE are different from cell to cell.

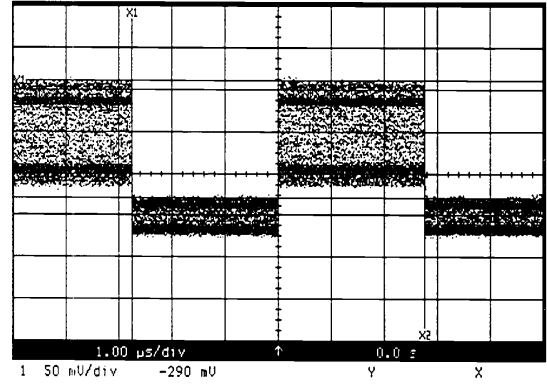

Fig. $7 \Delta G=4.0 \mathrm{~dB}$, injection current from 150 $\mathrm{mA}$ to $100 \mathrm{~mA}$ at input power of $-30 \mathrm{dBm}$

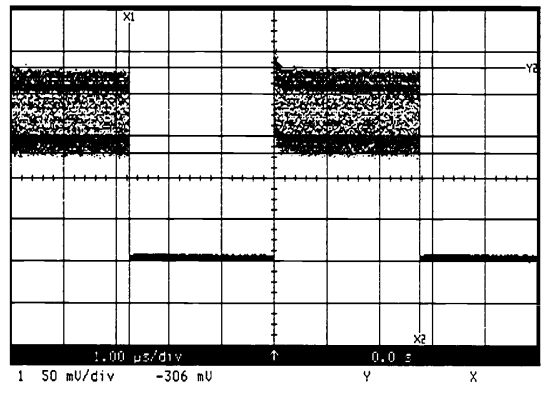

Fig. 8 SOA switched on/off at injection current $100 \mathrm{~mA}$ with input power of $-30 \mathrm{dBm}$, no ASE power contribution if SOA is off

Fig. 8 shows that a SOA is switched on/off with an injection current of $100 \mathrm{~mA}$, and with an input power of $-30 \mathrm{dBm}$. There is no ASE power contribution if the SOA is switched off. Fig.9 shows the first bytes of an amplified ATM packet through SOA, which is switched at the cell rate of $2.7 \mu$ s at 155.52 Mbps. In the experiment, an overhead of the cell is set as " 00111010 ", in which the first two " 00 " is taken as the guard time, and " 111 " is used for a power adjustment. From the measurement result, one can see that all the bits of "111" in the overhead are observed. There is a trade-off between the fast rise time and overshoot. In the example given, the rise time is $8.9 \mathrm{~ns}$ with an reasonable overshoot at the injection current of $100 \mathrm{~mA}$

Another important parameter is differential turn on delay, which is shown in Fig. 10. Here the turn on delay at injection current $150 \mathrm{~mA}$ is taken as a reference, the differential turn on delay is $4.7 \mathrm{~ns}$ when the injection current varies from $150 \mathrm{~mA}$ to $30 \mathrm{~mA}$. 


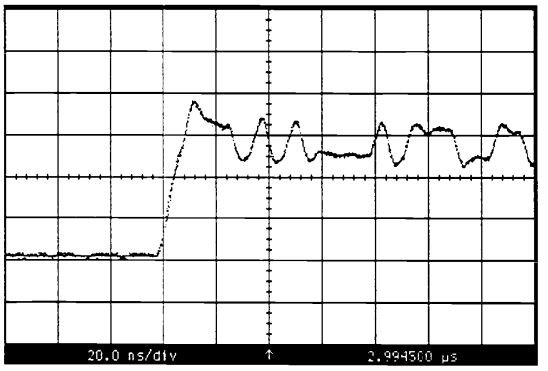

$20 \mathrm{~ns} / \mathrm{div}, 400 \mathrm{mv} / \mathrm{div}$

Fig.9 Amplified packet through a switched SOA

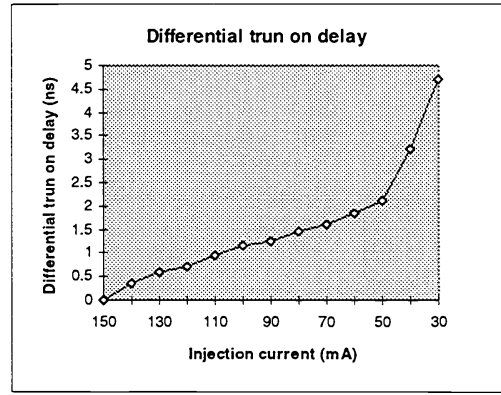

Fig.10 Differential turn on delay

\section{CONCLUSION}

By employing fast switching and accurate gain control, the problem caused by ASE noise accumulation from a number of SOAs operating in parallel is solved, and meanwhile the signal dynamic range is compressed. According to the risetime, and differential turn on delay of the measurement results, the burst mode switching of the SOA can be accomplished within 3 bits at $155.52 \mathrm{Mbps}$. A gain control range of the SOA larger than $10 \mathrm{~dB}$ is obtained by setting different values of the injection currents from $50 \mathrm{~mA}$ to 150 $\mathrm{mA}$.

\section{ACKNOWLEDGEMENT}

This work is supported by the European Commission within ACTS-PLANET project. Authors would like to thank all partners involved for their coorporation.

\section{REFERENCE}

[1] Ken-ichi Yukimatsu, Yoshihiro Shimazu, and Atsushi Hiramatsul, " A Photonic ATM Backbone Network" IEEE ICC'94, pp.1346-1351, May1-5, 1994, USA

[2] Denis J.G.Mestdagh and Claire M.Martin, “The Super-PON Concept and Its Technical Challenges”, IFIP/IEEE Broadband Communications'96, Apr.23-25,1996.

[3] Ingrid Van de Voorde, "PLANET: Photonic Local Access Network”, workshop on "Access Networks \& Related Components”, Brussels, Belgium, June 5, 1996.

[4] L.F.Tiemeijer, "High Performance MQW Laser Amplifiers for Transmission Systems Operating in the $1310 \mathrm{~nm}$ Window at Bitrates of $10 \mathrm{Gbit} / \mathrm{s}$ and Beyond”, ECOC'95, 21st European Conference on Optical Communication, Brussels, Belgium, 1995.

[5] N.A. Olsson, "Lightwave Systems With Optical Amplifiers", Journal of Lightwave Technology, Vol.7, No. 7, July, 1989.

[6] I. Van de Voorde, et.al, "Evaluation of network topologies for SuperPON access networks", NOC'96, Heidelberg, Germany, 1996. 